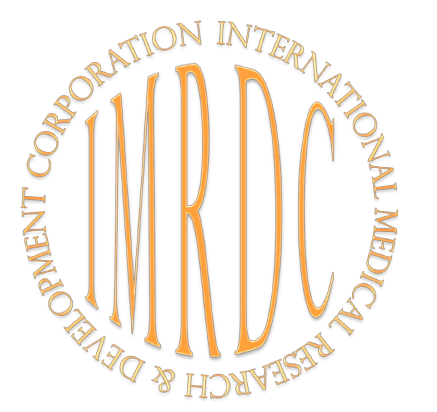

\title{
The Effectiveness of Laser Treatment for Diabetic Retinopathy in Patients with Chronic Kidney Disease
}

\author{
Maria N. Ponomareva ${ }^{1}, \mathrm{PhD}, \mathrm{ScD}$; Ivan M. Petrov ${ }^{1}, \mathrm{PhD}, \mathrm{ScD}$; \\ Evgeniya K. Gribanova ${ }^{2}$ \\ ${ }^{1} T y u m e n$ State Medical University \\ ${ }^{2}$ Multifunctional Clinical Diagnostic Center \\ Tyumen, the Russian Federation
}

\begin{abstract}
Background: Panretinal photocoagulation (PRP) remains one of the effective methods of treatment in pre- and proliferative forms of retinopathy with high efficiency. The aim of this study was to investigate the efficacy of PRP depending on the somatic status, laboratory parameters, and the severity of chronic kidney disease (CKD) in patients with type 2 diabetes (T2D) and a history of diabetic retinopathy (DR).

Methods and Results: The study included 76 patients (50 women and 26 men) with T2D who underwent PRP for DR (152 eyes) using a VISULAS ${ }^{\circledR} 532 \mathrm{~s}$ solid-state laser (ZEISS). The patients were divided into two groups depending on the severity of CKD. Group 1 ( $\mathrm{n}=32,64$ eyes) included patients with CKD Stage 1, Group 2 ( $\mathrm{n}=44,88$ eyes) included patients with CKD Stage 2. All patients underwent standard ophthalmological examination: visometry, tonometry, perimetry, biomicroscopy of the anterior segment of the eye and vitreous body, and fundus ophthalmoscopy. Thickness map of the retina was obtained using the RTVue-100 OCT (Optovue, Fremont, CA) EMM5 scan protocol and the Stratus OCT (Carl Zeiss Meditec, USA) radial scan protocol. Laboratory methods included a general blood test, PPG, FG, HbA1c, general urine analysis, and the assessment of blood levels of creatinine, ALT, and AST.

PRP was carried out according to the standard method, gradually, in three stages; the interval between the stages of laser treatment was 1 month. After laser treatment, all patients, regardless of the treatment stage, were prescribed topical Broxinac $\mathbb{R}$ (Bromfenac ophthalmic solution $0.09 \%$ ). The dynamics of corrected visual acuity (CVA) parameters and the retinal thickness of the macular region were assessed before PRP and 3 months after the complex treatment.

Multivariate analysis revealed a linear and nonlinear effect of lipid spectrum indicators on the formation of CL (crystalline lens) pathology. After treatment, a significant increase in CVA was noted in both study groups. The effectiveness of PRP coagulation depended on the severity of the CKD stage in T2D patients with DR.

Normalization of morphometric parameters of the macular region of the retina was noted in $93.8 \%$ of cases in Group 1 and in $86.4 \%$ of cases in Group 2. The decrease in the effectiveness of treatment was associated with the presence of macroangiopathy (coronary artery disease), concomitant diseases (chronic heart failure, hypertension and dyslipidemia), and CKD stage.

Conclusion: Prolonged administration of the non-steroidal, anti-inflammatory drug Bromfenacum ${ }^{\circledR}$ for a month after each stage of PRP is effective.(International Journal of Biomedicine. 2021;11(4):441-445.)
\end{abstract}

Key Words: diabetic retinopathy $\bullet$ chronic kidney disease $\bullet$ panretinal photocoagulation

For citation: Ponomareva MN, Petrov IM, Gribanova EK. The Effectiveness of Laser Treatment for Diabetic Retinopathy in Patients with Chronic Kidney Disease. International Journal of Biomedicine. 2021;11(4):441-445. doi:10.21103/Article11(4)_OA8

\section{Abbreviations}

AST, aspartate transaminase; ALT, alanine transaminase; BMI, body mass index; CKD, chronic kidney disease; CAD, coronary artery disease; CL, crystalline lens; CHF, chronic heart failure; CVA, corrected visual acuity; DR, diabetic retinopathy; DME, diabetic macular edema; GFR, glomerular filtration rate; HDL, high-density lipoprotein; LDL, low-density lipoprotein; PRP, panretinal photocoagulation; T2D, type 2 diabetes; TC, total cholesterol. 


\section{Introduction}

Diabetic retinopathy (DR) and diabetic nephropathy are typical microvascular complications of T2D caused by chronic hyperglycemia. ${ }^{(1-4)}$ Prevalence of CKD and DR increases proportionally to the disease duration in T2D..$^{(5-7)}$

DR associated with visual impairment has a significant impact on health-related quality of life. ${ }^{(8)}$ To treat early diabetic neovascular retinopathy, Beetham et al. in 1969 applied the first ruby laser photocoagulation. ${ }^{(9)}$ To date, the methods of retinal laser photocoagulation continue to be improved; the indications and contraindications for this type of treatment are being formed. ${ }^{(10)}$ PRP remains one of the effective methods of treatment in pre- and proliferative forms of retinopathy with high efficiency. ${ }^{(11,12)}$ The effectiveness of PRP varies from $60 \%$ to $99 \%$ and depends on the degree of suppression of vascularization, stabilization, and improvement of visual functions. ${ }^{(13,14)}$ Many researchers associate such a range of efficacy with the influence of somatic factors, significant fluctuations in laboratory parameters, and the presence of diabetic macular edema, which remains the main cause of vision loss in patients with DR. ${ }^{(10.15,16)}$

The aim of this study was to investigate the efficacy of PRP depending on the somatic status, laboratory parameters, and the severity of CKD in patients with T2D and a history of DR.

\section{Materials and Methods}

The study included 76 patients (50 women and 26 men) with T2D who underwent PRP for DR (152 eyes) using a VISULAS ${ }^{\circledR} 532 \mathrm{~s}$ solid-state laser (ZEISS).

The patients were divided into two groups depending on the severity of CKD. Group 1 ( $\mathrm{n}=32,64$ eyes) included patients with CKD Stage 1, Group 2 ( $\mathrm{n}=44,88$ eyes) included patients with CKD Stage 2.

In this study, the DR classification proposed by Kohner and Porta (1992) was used. The median age of the patients was $62[30 ; 77]$ years.

The exclusion criteria were T1D, the presence of the inflammatory, post-traumatic, and dystrophic diseases of the eyeball not associated with DM, as well as hereditary and congenital eye pathologies, CKD Stage 3.

All patients underwent comprehensive clinical examination. All patients were examined by a neurologist, therapist, endocrinologist, cardiologist, and podiatrist. CKD was diagnosed with determination of the blood creatinine level and further calculation of the GFR using the Cockcroft\&Gault formula.

All patients underwent standard ophthalmological examination: visometry, tonometry using non-contact pneumotonometer (Reichert Technologies), perimetry using PNR-2-01, biomicroscopy of the anterior segment of the eye and vitreous body on an SL-140 slit lamp (Carl Zeiss Meditec AG, Germany), and fundus ophthalmoscopy using a noncontact Ocular MaxField High Mag 78D Lens.

Thickness map of the retina was obtained using the RTVue-100 OCT (Optovue, Fremont, CA) EMM5 scan protocol and the Stratus OCT (Carl Zeiss Meditec, USA) radial scan protocol.

Laboratory methods included a general blood test, PPG, $\mathrm{FG}, \mathrm{HbAlc}$, general urine analysis, and the assessment of blood levels of creatinine, ALT, and AST.

PRP was carried out according to the standard method, gradually, in three stages; the interval between the stages of laser treatment was 1 month. After laser treatment, all patients, regardless of the treatment stage, were prescribed topical Broxinac ${ }^{\circledR}$ (Bromfenac ophthalmic solution 0.09\%). The dynamics of CVA parameters and the retinal thickness of the macular region were assessed before PRP and 3 months after the complex treatment.

The study was approved by local ethics committee, and written informed consent was obtained from all participants.

Statistical analysis was performed using IBM SPSS Statistics for Windows, Version 23.0. Armonk, NY: IBM Corp. The normality of distribution of continuous variables was tested by one-sample Kolmogorov-Smirnov test. Continuous variables with normal distribution were presented as mean (standard error of the mean $[\mathrm{SEM}]$ ); non-normal variables were reported as median (interquartile range (IQR; 25th to 75th percentiles). Student's unpaired and paired t-tests were used to compare two groups for data with normal distribution. MannWhitney U test was used to compare means of 2 groups of variables not normally distributed. The Wilcoxon criterion was used to compare the differences between the paired samples. The results are graphically presented in the form of a Box and Whisker Plot. A value of $P<0.05$ was considered significant.

\section{Results}

Comparative characteristics of clinical and laboratory parameters (Table 1) revealed significant differences in the study groups by age $(P=0.025)$, duration of the disease $(P=0.015)$, and GFR $(P=0.015)$. The groups did not differ in laboratory parameters of the lipid profile, FG, PPG, HbAlc, and liver function tests (Table 1).

After the combined treatment, there was a significant increase in CVA in both groups: from $0.60(0.2 ; 1.0)$ to $0.69(0.3 ; 1.0)(P=0.000)$ in Group 1 and from $0.53(0.04 ; 1.0)$ to $0.63(0.04 ; 1.0)(P=0.000)$ in Group 2.

The quality of vision and visual prognosis in patients of both groups was influenced by the CL transparency and the state of the macular region of the retina (Table 2). In Group 1, every second patient had CL pathology, and 12.5\% of patients had pseudophakia. In Group 2, the incidence of the CL pathology was $77.3 \%$, including pseudophakia in $27.3 \%$ of patients, which had a positive effect on visual acuity indicators. Thus, in Group 1, patients with posterior chamber intraocular lenses accounted for $12.5 \%$ (6.25\% women, $6.25 \%$ men); in Group 2, this indicator was 2 times higher - $27.26 \%$ (11.36\% women, $15.9 \%$ men).

Multivariate analysis revealed a linear and nonlinear effect of lipid spectrum indicators (TC and LDL) on the formation of CL pathology, including in patients who received surgical treatment (Fig.1). BMI and HDL indices on their own did not have a reliably significant effect on CL pathology. 
Table 1.

Characteristics of clinical and laboratory parameters in study groups

\begin{tabular}{|l|c|c|c|}
\hline \multicolumn{1}{|c|}{ Variable } & $\begin{array}{c}\text { Group 1 } \\
(\mathrm{n}=32)\end{array}$ & $\begin{array}{c}\text { Group } 2 \\
(\mathrm{n}=44)\end{array}$ & $P$-value \\
\hline Age, yrs & $59.81 \pm 9.88$ & $64.14 \pm 6.60$ & 0.025 \\
\hline Gender, M/F & $20 / 12$ & $30 / 14$ & 0.606 \\
\hline Disease duration, yrs & $12.06 \pm 5.99$ & $15.55 \pm 6.14$ & 0.015 \\
\hline BMI, kg/m² & $31.63 \pm 5.92$ & $30.36 \pm 5.30$ & 0.33 \\
\hline TC, mmol/L & $5.00 \pm 1.17$ & $4.65 \pm 1.1$ & 0.183 \\
\hline LDL, mmol/L & $3.09 \pm 1.04$ & $3.0 \pm 0.94$ & 0.685 \\
\hline HDL, mmol/L & $1.04 \pm 0.66$ & $1.35 \pm 0.49$ & 0.676 \\
\hline AST, U/L & $22.07 \pm 10.54$ & $24.16 \pm 18.49$ & 0.566 \\
\hline ALT, U/L & $25.54 \pm 10.54$ & $24.52 \pm 16.85$ & 0.772 \\
\hline FG, mmol/L & $9.29 \pm 3.17$ & $8.82 \pm 3.59$ & 0.483 \\
\hline PPG, mmol/L & $13.64 \pm 3.88$ & $12.96 \pm 2.67$ & 0.365 \\
\hline HbA1c, \% & $8.87 \pm 1.83$ & $9.39 \pm 1.65$ & 1.199 \\
\hline GFR, ml/min/1.73m ${ }^{2}$ & $98.34 \pm 3.19$ & $73.21 \pm 0.00$ & 0.000 \\
\hline
\end{tabular}

Table 2.

The structure of the CL pathology in the study groups

\begin{tabular}{|c|c|c|c|}
\hline Pathological condition & $\begin{array}{c}\text { Group 1 } \\
(\mathrm{n}=64)\end{array}$ & $\begin{array}{c}\text { Group 2 } \\
(\mathrm{n}=88)\end{array}$ & $P$-value \\
\hline Without CL pathology & $32(50.0 \%)$ & $20(22.7 \%)$ & \\
Male & $\begin{array}{c}16(25.0 \%) \\
\text { Female }\end{array}$ & $\begin{array}{c}8(9.1 \%) \\
16(25.0 \%)\end{array}$ & 0.000 \\
\hline CL pathology & $32(50 \%)$ & $68(77.3 \%)$ & 0.000 \\
\hline Cataract, & $24(37.5 \%)$ & $44(50.0 \%)$ & \\
Male & $\begin{array}{c}4(6.25 \%) \\
\text { Fmale }\end{array}$ & $\begin{array}{c}6(6.8 \%) \\
19(43.2 \%)\end{array}$ & 0.126 \\
\hline Pseudophakia & $8(12.5 \%)$ & $24(27.3 \%)$ & \\
Male & $\begin{array}{c}4(6.25 \%) \\
4(6.25 \%)\end{array}$ & $\begin{array}{l}14(15.9 \%) \\
10(11.4 \%)\end{array}$ & 0.027 \\
\hline Female & & & \\
\hline
\end{tabular}

The study showed a more pronounced incidence of CL pathology in patients of Group 2 and revealed a significant effect of age $(\mathrm{r}=0.4, P=0.045)$, disease duration $(\mathrm{r}=0.5$, $P=0.017)$ and GFR $(\mathrm{r}=0.6, P=0.016)$.

Morphometric parameters of the perimacular and macular areas of the retina did not reveal significant differences between the groups before PRP. After the full course of treatment, a significant decrease in the retinal thickness was noted in the nasal $(P=0.015)$ (Fig. 2), temporal $(P=0.041)$, and inferior segments $(P=0.044)$. There were no negative dynamics in CVA and morphometric parameters of the retina against the background of combined treatment (PRP and Bromfenac ophthalmic solution $0.09 \%$ ) in patients with CKD Stages 1 and 2.

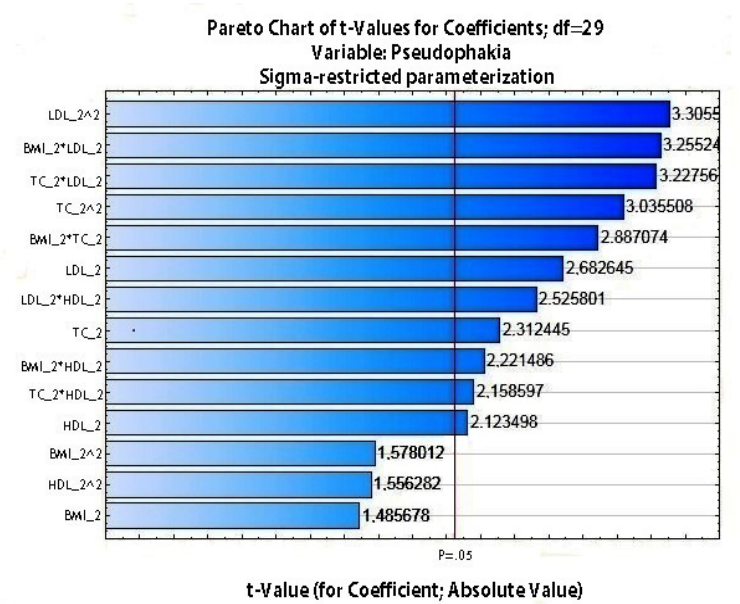

Fig. 1. Multivariate analysis. Effects of lipid spectrum indicators on the formation of CL pathology, including in patients who received intraocular lens implantation surgery.

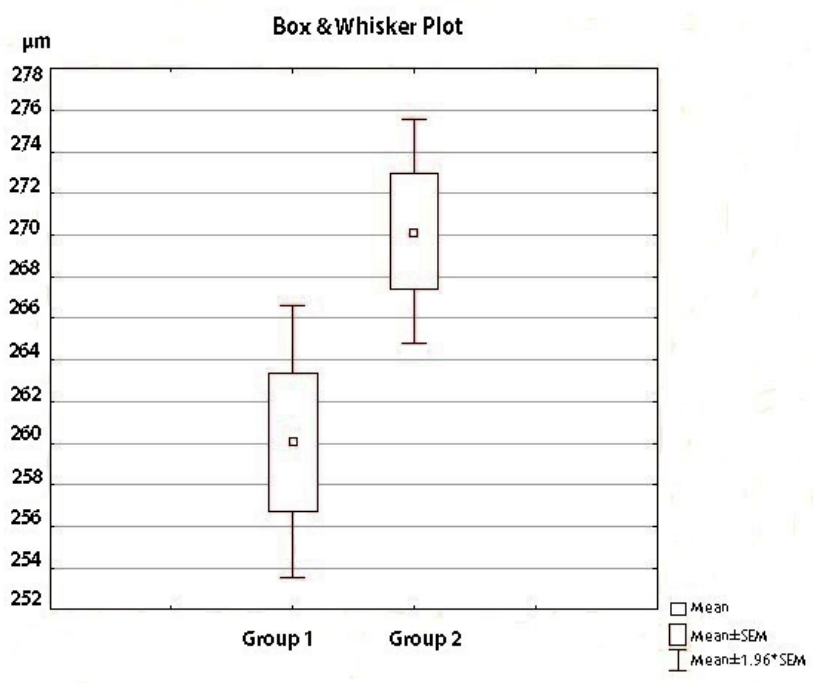

Fig. 2. Retinal thickness $(\mu m)$ in the nasal segment after the full course of treatment in the study groups.

In Group 1, DME in both eyes was diagnosed in $25 \%$ of patients $(12.5 \%$ of women, $87.5 \%$ of men) with disease duration of $12.75 \pm 4.9$ years. In Group 2, DME was observed in $34.1 \%$ of patients $(46.2 \%$ of women and $53.8 \%$ of men) with disease duration of $18.38 \pm 4.1$ years.

Diabetic macroangiopathies in patients with DME were represented only by coronary artery disease. In Group 1 , concomitant diseases were represented by dyslipidemia 
$(75 \%)$, obesity $(75 \%)$, arterial hypertension $(62.5 \%)$, and chronic heart failure (37.5\%). In Group 2, obesity, arterial hypertension, and dyslipidemia were encountered with the same frequency ( $92.3 \%$ of cases), and chronic heart failure in $69.2 \%$ of cases

In Group 1, during treatment, complete normalization of the retinal thickness was achieved in $6(75.0 \%)$ patients; DME remained in the temporal and superior segments in $2(25.0 \%)$ patients. In Group 2, there was a tendency towards a decrease in retinal thickness in the temporal and superior segments, and in $9(60.0 \%)$ patients, retinal thickness in the nasal, inferior, and central segments was normalized. Analysis of the somatic status of patients with incomplete regression of DME revealed the presence of coronary artery disease, chronic heart failure and dyslipidemia, which can be considered as predictors for DME in DR patients against the background of CKD Stages 1 and 2.

\section{Discussion}

Domestic and foreign scientists have demonstrated the effect of the somatic status features on the development and severity of DR and visual prognosis. ${ }^{(2,4,10)}$ In our study, CVA depended on the CL transparency and the morphometric parameters of the macular region. Our results show a significant effect of the CKD stage, somatic status (patient's age, disease duration, body mass index), laboratory parameters (total cholesterol, low-density lipoprotein) on CL opacity in T2D patients. However, in both study groups, there were patients who underwent surgery to replace the cloudy lens with an intraocular lens, which could affect the CVA values. In Group 2, CL pathology occurred by $27.3 \%$ more often than in Group 1. Thus, CKD is a trigger for CL opacity, which depends on the severity of kidney pathology. These data require further clinical research. Against the background of topical use of the non-steroidal, anti-inflammatory drug Bromfenacum ${ }^{\circledR}$ 0.09\%(for a month after each stage of treatment), PRP was shown to be effective in $93.8 \%$ of patients in Group 1 and in $86.4 \%$ of patients in Group 2. In DME, the normalization of morphometric parameters of the macular region of the retina was influenced by the presence of macroangiopathies, concomitant diseases, and the CKD stage.

Thus, macroangiopathies, concomitant diseases, and the CKD stage can also be attributed to biological markers for the DME development. It is necessary to continue monitoring patients with incomplete regression of DME and further correction of the scheme for topical and systemic treatment with normalization of the somatic status in order to restore the morphometric integrity of the retina and reduce the risk for the progression of retinal proliferative processes.

\section{Conclusion}

Based on the monitoring of clinical and functional indicators, a significant increase in CVA was noted in both study groups. Analysis of the medical history, somatic status, and laboratory parameters helps to identify biological markers of CL opacity.

The effectiveness of PRP coagulation depends on the severity of the CKD stage in T2D patients with DR.

Normalization of morphometric parameters of the macular region of the retina was noted in $93.8 \%$ of cases in Group 1 and in $86.4 \%$ of cases in Group 2. The decrease in the effectiveness of treatment was associated with the presence of macroangiopathy (coronary artery disease), concomitant diseases (chronic heart failure, hypertension and dyslipidemia), and CKD stage.

Prolonged administration of the non-steroidal, antiinflammatory drug Bromfenacum ${ }^{\circledR}$ for a month after each stage of PRP is effective.

\section{Competing Interests} interests.

The authors declare that they have no competing

\section{References}

1. Papatheodorou K, Papanas N, Banach M, Papazoglou D, Edmonds M. Complications of Diabetes 2016. J Diabetes Res. 2016;2016:6989453. doi: 10.1155/2016/6989453.

2. Khalil SA, Megallaa MH, Rohoma KH, Guindy MA, Zaki A, Hassanein M, Malaty AH, Ismael HM, Kharboush IF, El Kafash DN, Sallam HN, Desouky IA. Prevalence of Chronic Diabetic Complications in Newly Diagnosed versus Known Type 2 Diabetic Subjects in a Sample of Alexandria Population, Egypt. Curr Diabetes Rev. 2019;15(1):74-83. doi: 10.2174/1573399814666180125100917.

3. Wang W, He M, Gong X, Wang L, Meng J, Li Y, Xiong $\mathrm{K}, \mathrm{Li}$ W, Huang W. Association of renal function with retinal vessel density in patients with type 2 diabetes by using sweptsource optical coherence tomographic angiography. $\mathrm{Br} \mathrm{J}$ Ophthalmol. 2020 Dec;104(12):1768-1773. doi: 10.1136/ bjophthalmol-2019-315450.

4. Xu X, Gao B, Ding W, Wang Q, Zhang M, Tan T, Sun F, Lei J, Ji Q, Xu F. Retinal image measurements and their association with chronic kidney disease in Chinese patients with type 2 diabetes: the NCD study. Acta Diabetol. 2021 Mar;58(3):363-370. doi: 10.1007/s00592-020-01621-6.

5. Adler AI, Stevens RJ, Manley SE, Bilous RW, Cull CA, Holman RR; UKPDS GROUP. Development and progression of nephropathy in type 2 diabetes: the United Kingdom Prospective Diabetes Study (UKPDS 64). Kidney Int. 2003 Jan;63(1):225-32. doi: 10.1046/j.15231755.2003.00712.x.

6. Klein R, Klein BE, Moss SE, Davis MD, DeMets DL. The Wisconsin epidemiologic study of diabetic retinopathy. III. Prevalence and risk of diabetic retinopathy when age at diagnosis is 30 or more years. Arch Ophthalmol. 1984 Apr;102(4):52732. doi: 10.1001/archopht.1984.01040030405011.

\footnotetext{
*Corresponding author: Dr. Evgeniya K. Gribanova. Multifunctional Clinical Diagnostic Center, Tyumen, the Russian Federation.E-mail:gribanova.1988@inbox.ru
} 
7. Park HC, Lee YK, Cho A, Han CH, Noh JW, Shin YJ, Bae SH, Kim H. Diabetic retinopathy is a prognostic factor for progression of chronic kidney disease in the patients with type 2 diabetes mellitus. PLoS One. 2019 Jul 29;14(7):e0220506. doi: 10.1371/journal.pone.0220506.

8. Coyne KS, Margolis MK, Kennedy-Martin T, Baker TM, Klein R, Paul MD, Revicki DA. The impact of diabetic retinopathy: perspectives from patient focus groups. Fam Pract. 2004 Aug;21(4):447-53. doi: 10.1093/fampra/ $\mathrm{cmh} 417$.

9. Beetham WP, Aiello LM, Balodimos MC, Koncz L. Ruby-laser photocoagulation of early diabetic neovascular retinopathy: preliminary report of a long-term controlled study. Trans Am Ophthalmol Soc. 1969;67:39-67.

10. Standards of specialized diabetes care. Edited by Dedov I.I., Shestakova M.V., Mayorov A.Yu. 9th edition. Sakharni Diabet. 2019;22(1S1):1-144. [In Russian].

11. Bobykin EV. [Current approaches to the treatment of diabetic macular edema. A literature review]. Fyodorov Journal of Ophthalmic Surgery. 2019;(1):67-76. doi: 10.25276/0235-
4160-2019-1-67-76. [Article in Russian].

12. Mozherencov VP, Prokofyeva GL, Usova LA. [Ocular manifestations of diabetes mellitus]. Russian Journal of Clinical Ophthalmology. 2002;1:31. [Article in Russian].

13. PalankerD, BlumenkranzMS. Panretinal photocoagulation for proliferative diabetic retinopathy. Am J Ophthalmol. 2012 Apr;153(4):780-1; author reply 781-2. doi: 10.1016/j. ajo.2012.01.001.

14. Distefano LN, Garcia-Arumi J, Martinez-Castillo V, Boixadera A. Combination of Anti-VEGF and Laser Photocoagulation for Diabetic Macular Edema: A Review. J Ophthalmol. 2017;2017:2407037. doi: $10.1155 / 2017 / 2407037$.

15. Browning DJ, Stewart MW, Lee C. Diabetic macular edema: Evidence-based management. Indian J Ophthalmol. 2018 Dec;66(12):1736-1750. doi: 10.4103/ijo. IJO 124018.

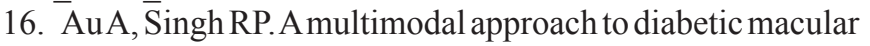
edema. J Diabetes Complications. 2016 Apr;30(3):545-53. doi: 10.1016/j.jdiacomp.2015.11.008. 